\title{
List of Conference Participants
}

Allard France: CRAL, France

Allen Michelle: University of New South Wales, Australia,

Allwood Abigail: Queensland University of Technology, Australia Andreeshchev Andrey: University of Texas, USA

Anitori Roberto: Australian Centre for Astrobiology, Australia

Ansbro Eamonn: Space Exploration Ltd, Ireland

Aspin Colin: Gemini North, USA

Bailey Jeremy: Anglo Australian Observatory, Australia

Bakes Emma: SETI Institute and NASA Ames, USA

Barnes Geoffrey: Massey University, New Zealand

Bauer Annette: Australian Centre for Astrobiology, Australia

Beare Richard: Warwick University, UK

Beichman Charles: NASA JPL, USA

Bhathal Ragbir: University of Western Sydney, Australia

Blair Jaime: Pennsylvania State University, USA

Blumberg Baruch: NASA NAI, USA

Bonaccorsi Rosalba: University of Trieste, Italy

Brodrick David: Australia Telescope National Facility, Australia

Brown Adrian: Australian Centre for Astrobiology, Australia

Bryden Geoffrey: NASA JPL, USA

Burton Michael: University of New South Wales, Australia

Caldwell Douglas: SETI Institute and NASA, USA

Campbell John: James Cook University, Australia,

Carrigan Dick: Fermi National Laboratory, USA

Catling David: University of Washington, USA

Chaisson Eric: Tufts University, USA

Chamberlain Sarah: Macquarie University and AAO, Australia

Chiar Jean: SETI Institute and NASA, USA

Christian Carol: Space Telescope Science Inst, USA

Chyba Chris: SETI Institute, USA

Clampin Mark: Space Telescope Institute, USA

Clarke James: Swinburne University, Australia

Clayton Geoffrey: Lousiana State University, USA

Connard Phillip: Australia

Conway-Morris Simon: Cambridge University, UK

Coustenis Athena: Meudon Observatory, France

Cullers Kent: SETI Institute, USA

Daniel Scott: Australia

Davies Paul: Australian Centre for Astrobiology, Australia

Demorest Paul: Space Science Laboratory, UC Berkeley, USA

DeVore Edna: SETI Institute, USA

Dopatka Ulrich: University Bern, Switzerland

Doyle Laurance, SETI Institute, USA

Drake Frank: SETI Institute, USA

Dreher John: SETI Institute, USA 
Ekers Ron: Australia Telescope National Facility, Australia

Finley Jason: UCLA, USA

Fisher Jane: SETI Institute, USA

Flynn George: State University of New York, USA

Franck Siegfried: Potsdam Institute of Climate Impact Research, Germany

Friedland Peter: USA

Fujikawa Shelly: Harvard University, USA

Fulton Steven: Space Science Laboratory, UC Berkeley, USA

Gaidos Eric: University of Hawaii, USA

Gargaud Muriel: Observatoire de Bordeaux, France

Gibson Morgan: NASA JSC, USA

Gibson Everett: NASA JSC, USA

Gilmour Iain: Open University, UK

Grashuis Randy: Gemini South, Chile

Griffiths Martin: University of Glamorgan, UK

Grymes Rose: NASA Astrobiology Institute, USA

Guogas Laura: Harvard University, USA

Hanczyc Martin: Harvard University, USA

Harrison Mark: Australian National University, Australia

Haywarden Timothy: Royal Observatory, Scotland

Heacox William: University of Hawaii, USA

Hedges Blair: Pennsylvania State University, USA

Helo Erkki: University of Helsinki, Finland

Hidas Marton: Unviersity of New South Wales, Australia

Hill Andrew: Australian Centre for Astrobiology, Australia

Hogbom Jan: Stockholm Observatory, Sweden

Hood Ben: University of Arkansas, USA

Hough James: University of Hertfordshire, UK

Howard Andrew: Harvard University, USA

Hunt Maria: University of new South Wales, Australia

Jayawardhana Ray: University of California, Berkeley, USA

Jenkins Alan: USA

Jenniskens Peter: NASA Ames, USA

Johnson Mark: University of Western Sydney, Australia

Johnston Ray: The Great Barrier Reef Observatory, Australia

Jones Paul: Australia Telescope National Facility, Australia

Jugaku Jun: Research Institute of Civilisation, Japan

Junge Karen: University of Washington, USA

Kaufmann Michael: Universitat Witten, Germany

Kay Laura: Columbia University, USA

Keller Horst Uwe: Max Planck Institute, Germany

Kennedy Tim: Australia Telescope National Facility, Australia

Khare Bishun: SETI Institute and NASA Ames, USA

Knight Sue: Sutherland Astronomical Society, Australia

Koch David: NASA Ames, USA

Koeberl Christian: University of Vienna, Austria

Korpela Eric: Space Science Laboratory, UC Berkeley, USA 
Kral Tim: University of Arkansas, USA

Kuan Yi-Jehng: National Taiwan Normal University, Taiwan

Kwok Sun: University of Calgary, Canada

Laing Jennifer: Mars Society, Australia

Lazcano Antonio: Universidad Nacional Autonoma de Mexico, Mexico

Lazio Joseph: Naval Research Laboratory, USA

Le Pevelen Delphine: Imperial College, London, UK

LeMarchand Guillermo: , Argentina

Levasseur-Regourd AnnyChantal: University Paris VI/Aeronomie, France

Lineweaver Charles: University of New South Wales, Australia

Lucas Philip: University of Hertfordshire, UK

Maddison Sarah: Swinburne University, Australia

Mandell Avram: Pennsylvania State University, USA

Marcy Geoff: University of California Berkeley, USA

Marino Lori: Emory University, USA

Marov Mikhail: Russian Academy of Sciences, Russia

Martin Eduardo: University of Hawaii, USA

Maurel Marie-Christine: Jacque-Monod University, France

McConnell Brian: Pacific Telephony Design, USA

McQuinley Matthew: Australia

McShea Daniel: Duke University, USA

Meadows Vikki: NASA JPL, USA

Meech Karen: University of Hawaii, USA

Methot Laura: UCLA, USA

Minard Robert: Pennsylvania State University, USA

Mix Lucas: Harvard University, USA

Mojzsis Stephen: University of Colorado, USA

Moon Darrel: Oxnard College, USA

Moore Lesa: Australian Centre for Astrobiology, Australia

Musso Paulo: Pontifical University, Italy

Neller Chris: SETI Institute, USA

Nemcek Milos: Technical University, Czech Republic

Newman Neal: NASA Representative for Australia, USA

Noll Keith: Space Telescope Science Institute, USA

Norris Ray: Australia Telescope National Facility, Australia

Oliver Carol: Australian Centre for Astrobiology, Australia

Ortolani Sergio: University of Padova, Italy

Overhauser Catherine: Space Exploration Ltd, Ireland

Paine Michael: Planetary Society, Australia

Papineau Dominic: University of Colorado, USA

Pearlstein Robert: IUPUI, USA

Perkins Karla: , Australia

Pierazzo Elizabeth: Planetary Science Institute, USA

Pittichova Jana: University of Hawaii, USA

Price Buford: UC Berkeley (Physics), USA

Quirrenbach Andreas: UC San Diego, USA

Race Margaret: SETI Institute, USA 
Rampino Michael: New York University, USA

Reid Iain Neill: Space Telescope Science Institute, USA

Roche Rose: CSIRO/University of Queensland, Australia

Ronimus Ron: University of Waikato, NZ

Ruiterkamp Richard: Leiden University, Netherlands

Runnegar Bruce: UCLA, USA

Sackett Penny: Mt Stromlo Observatory, Australia

Sallmen Shauna: UC Berkeley, USA

Schmitt Harrison: Apollo 17, USA

Schwartzman David: Howard University, USA

Selsis Franck: Centro de Astrobiologia, Spain

Shirai Toshimichi: Goldman Sachs, Japan

Shostak Seth: SETI Institute, USA

Siefert Janet: Rice University, USA

Sim Helen: Australia Telescope National Facility, Australia

Sogin Mitchell: Marine Laboratory, Woods Hole, USA

Soldan Jan: L'Universite de Geneve, Switzerland

Sparks Bill: Space Telescope Science Institute, USA

Stone Remington: Lick Observatory, USA

Stootman Frank: University of Western Sydney, Australia

Storrie-Lombardi Michael: NASA JPL, USA

Sutton Edmond: University of Ilinois, USA

Tabor Bruce: Australia

Tarter Jill: SETI Institute, USA

Taylor Russ: Australia Telescope National Faciltiy, Australia

Terrile Richard: NASA JPL, USA

Theison David: University of Maryland, USA

Thresher Wayne: NZ Institute for Crop and Food Research, New Zealand

Tinney Chris: Anglo Australian Observatory and SOC, Australia

Tough Allan: University of Toronto, Canada

Troncale Len: California State Polytechnic University, USA

Tsapin Alexandre: NASA JPL, USA

Vakoch Doug: SETI Institute, USA

Valtaoja Esko: Tuorla Observatory, Finland

Van Kranendonk Martin: Australian Centre for Astrobiology, Australia

Varki Ajit: UC San Diego, USA

Walter Malcolm: Australian Centre for Astrobiology, Australia

Welch Jack: UC Berkeley (Radio Astronomy), USA

Welsh William: San Diego State University, USA

Welstead Noel: SETI Research and Development Institute, Australia

West Michael: Mars Society, Australia

White Glenn: University of Kent, UK

Whittet Douglas: Rensselaer Polytechnic Institute, USA

Wolstencroft Ray: Royal Observatory, Scotland

Wright Shelley: Lick Observatory, USA

Zadnik Mario: Curtin University, Australia

Zinnecker Hans: Astrophysikalisches Institut Potsdam, Germany 
xvi

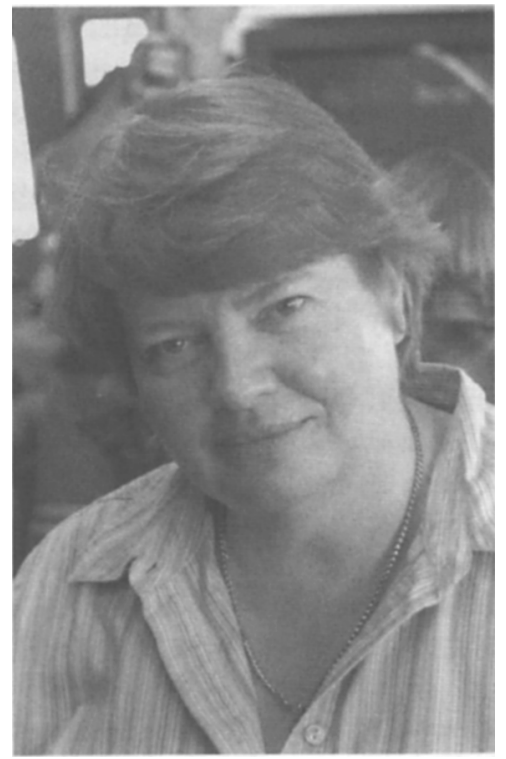

Carol Oliver, Chair of the LOC, without whom this symposium would not have happened (photo: Seth Shostak) 